\title{
Aménagements de la Centrale de Volhov en Russie
}

\author{
(SUITE ET FIN)
}

Pour le déplacement des transformateurs, on a installé un chariot transbordeur spécial qui roule sur une voie placée sur toute la longueur du bâtiment des vannes et au-dessus des appareils de manœurre des vannes d'amenée de l'eau aux turbines. Les transformateurs peuvent être ainsi transportés à l'atelier qui fait suite à la salle des machines principale. Cet atelier est équipé avec un pont roulant de 40 tonnes et contient les appareils nécessaires au filtrage et au séchage de l'huile, etc. où il peut être pris par le transbordeur des transformateurs et conduit à l'atelier de réparations.

De la salle des interrupteurs à huile, les conducteurs s'en vont vers la salle des sectionneurs située à l'étage supérieur, au niveau 38,50 .

La figure 8 montre comment est équipée cette salle. Les sectionneurs sont tripolaires du type normal à couteaux. Les volants de manceuvre ont été placés dans un couloir spécial

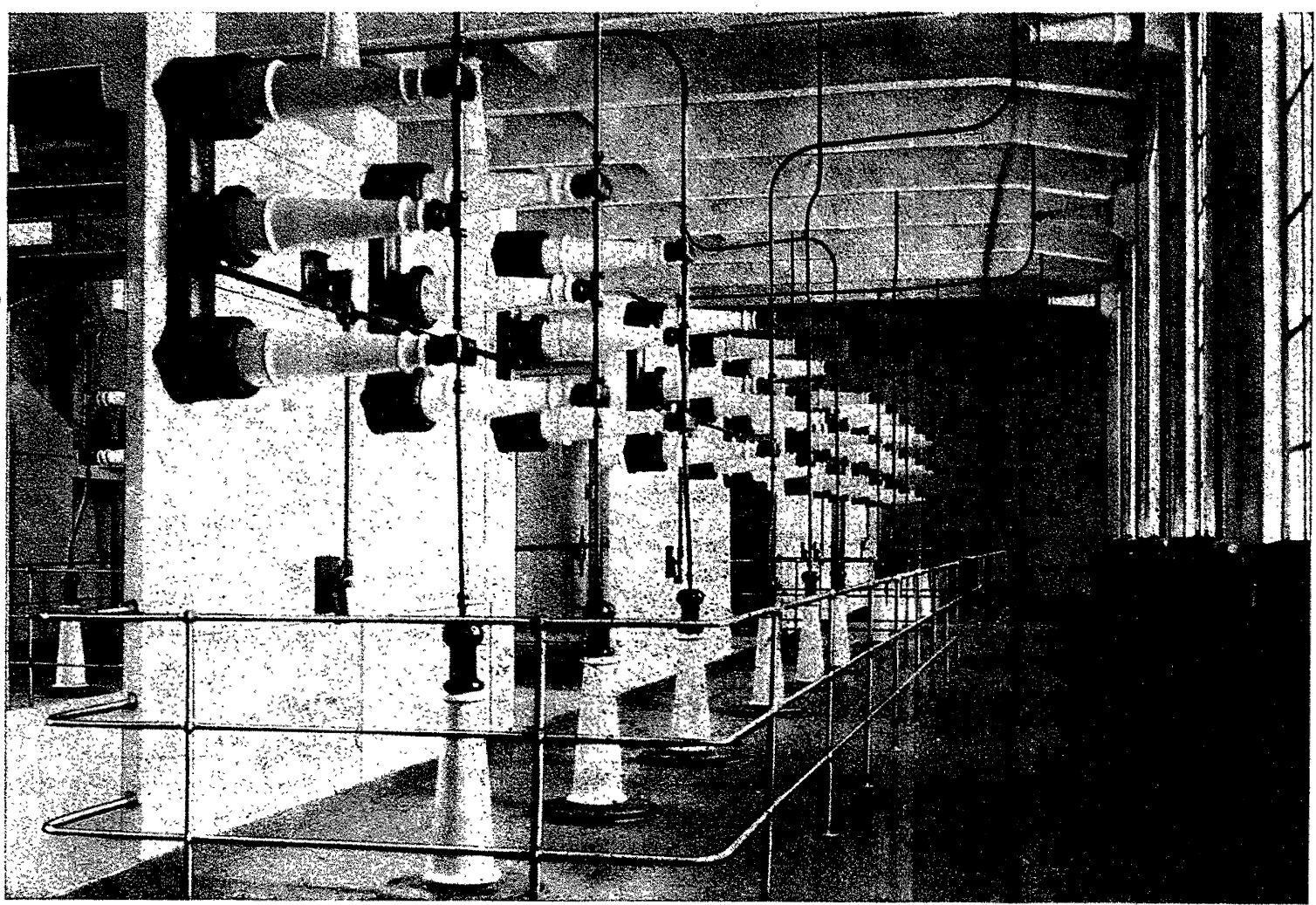

Fig. 8. - Sectionneur à $120 \mathrm{kV}$.

Des transformateurs, les conducteurs gagnent l'appareillage à $120 \mathrm{kV}$. (fig. 7) qui est monté dans deux tours (voir fig. 1), l'une à l'extrémité nord, l'autre à l'extrémité sud. Ces tours complètent fort bien le bâtiment d'appareillage. Chacune des tours a deux étages; le premier, au niveau 30,50 renferme les interrupteurs à huile à $120 \mathrm{kV}$. Les conducteurs haute tension consistent en tubes de cuivre de $38 \mathrm{~mm}$. de diamètre et $2 \mathrm{~mm}$. d'épaisseur. Dans la tour nord, il y a quatre interrupteurs à huile (fig. 7), deux d'entre eux pour les deux groupes de transformateurs nord, un autre pour la ligne qui part de cette tour et le dernier pour l'autre groupe de transformateurs et pour le raccordement des tours nord et sud.

La tour sud renferme trois interrupteurs à huile semblables, un interrupteurs de transformateur, un interrupteur de ligne et un interrupteur de sectionnement. Tous ces interrupteurs à huile peuvent supporter un courant de rupture de $15.000 \mathrm{amp}$., ils ont des chambres d'explosion et des transformateurs de courant qui font corps avec les isolateurs de traversée. Il y a un bac à huile séparé par pôle, la fermeture est faite électriquement par servo-moteur. Comme le montre la figure 7, tous les interrupteurs sont placés en ligne au-dessus des orifices d'évacuation de l'huile. Le chariot transbordeur est installé de façon que chaque pôle puisse être enlevé séparément, amené au monte-charge dont nous avons parlé précédemment et descendu à l'étage 30,50 qui renferme non seulement les volants correspondants aux sectionneurs, mais encore les dispositifs de manceuvre des interrupteurs à huile situés à l'étage supérieur. Ces dispositions offrent la plus grande sécurité pour le personnel et empêchent toute fausse manœuvre. Tous les sectionneurs et conducteurs sont fort bien protégés : il est possible de procéder à une visite du matériel sans courir aucun risque.

Les salles de transformateurs, d'interrupteurs et de sectionneurs sont séparées l'une de l'autre par des cloisons à l'épreuve du feu. Tous les conducteurs qui traversent les murs, les planchers et les plafonds, sont placés dans des isolateurs de traversée ; tout danger d'incendie est ainsi éliminé. Chaque transformateur a un sectionneur placé entre l'interrupteur à huile et les barres omnibus; les interrupteurs de sectionnement et les interrupteurs de ligne ont un double jeu

de sectionneurs. Ces derniers ont encore, en outre, des sectionneurs de shuntage permettant de mettre chaque interrupteur de ligne hors service et hors tension, en vue d'une inspection, sans que la fourniture du courant soit interrompue, l'interrupteur de transformateur agissant alors comme interrupteur de ligne.

Les sectionneurs de départ sont directement raccordés aux bobines de self montées sur consoles en béton armé et connectées aux isolateurs de traversée de la ligne Sur cette ligne et immédiatement après les bobines de self, sont installés des interrupteurs monopolaires manœuvrés par volant et qui peuvent servir à la mise à la terre de la ligne, phase par phase. Les isolateurs de sortie sont montés dans des niches ménagées dans les murs de la tour. A l'extérieur, au-dessus d'eux, on a placé un auvent. La ligne de transmission aboutit à ces isolateurs de sortie. Elle traverse immédiatement la rivière en une portée de 380 mètres et s'attache sur l'autre rive au premier pylone de la ligne aérienne.

Le câblage de signalisation des interrupteurs de sectionnement et les câbles des servo-moteurs des interrupteurs à huile sont placés dans un caniveau ménagé dans le plancher du cotloir de manœuvre et recouvert de plaques de béton.

De ce caniveau, ces câbles passent à un autre qui renferme également le petit câblage des interrupteurs et des transformateurs allant à la salle de contrôle. 
L'étage supérieur de la tour renferme les barres omnibus a $120 \mathrm{kV}$. qui sour entourées de murs de protection en béton (voir lig. 9). Une ligne extérieure tendue entre les deux tours établit une liaison entre les barres des tours sud et nord. Les détails de construction de cette ligne sont les mêmes que pour les deux lignes de transmission.

Services auxiliaires. Comme nous l'avons déjà dit, les alternateurs principaux ont des groupes moteurs-générateurs d'excitation séparés. Les moteurs sont alimentés par deux alternateurs auxiliaires d'une puissance unitaire de $1.250 \mathrm{kVA}$. installés au centre de la salle des machines et directement accouplés à des turbines hydrauliques de $1.400 \mathrm{HP}$. tournant à $150 \mathrm{t}$. p. m. Chacun de ces alternateurs peut, à lui seul, assurer la fourniture du courant d'excitation nécessaire à toute la centrale. L'appareillage de ces machines est placé au milieu de la centrale au niveau 11,80 , c'est-à-dire à l'étage quì se trouve immédiatement sous la salle des machines. Des alternateurs, les câbles partent vers deux interrupteurs à huile placés sur la charpente d'appareillage. Par l'intermédiaire des sectionneurs, ces interrupteurs peuvent être raccordés à un double jeu de barres omnibus désigné par la lettre A. Tous les circuits essentiels de manœuvre de la centrale sont raccordés à ces barres, notamment ceux des huit moteurs des excitatrices, du groupe d'excitation de réserve et des transformateurs des moteurs-générateurs pour le réglage automatique de la tension du système à $11 \mathrm{kV}$. Pour les services auxiliaires, il y a deux transformateurs, de $1.250 \mathrm{kVA}$. chacun, alimentés par les barres principales à $11 \mathrm{kV}$. et qui abaissent la tension à $2,2 \mathrm{kV}$. Ces transformateurs sont du type habituel triphasé à refroidissement par circulation d'eau.

Ils sont installés au niveau 16,20 dans un local compris entre les deux salles où se trouvent les boîtes terminales des câbles, à l'étage situé sous les interrupteurs principaux. Les transformateurs des services auxiliaires sont raccordés par câbles, du côté à $2,2 \mathrm{kV}$, à des transfórmateurs de réglage qui permettent une variation de tension de $\pm 7 \%$. Par l'intermédiaire d'inlerrupteurs et de sectionneurs, la connexion peut ètre rétablie à l'un des systèmes de barres $\mathrm{A}$ ou $\mathrm{B}$. La connexion normale est établie au système $\mathrm{B}$, qui est appelé le système local à $2,2 \mathrm{kV}$. Il en part seize circuits, qui alimentent un certain nombre de groupes auxiliaires, tels que ventilateurs et postes de charge de batterie, ponts roulants, pompes, petits transformateurs, abaisseurs de tension, etc. Des bobines de réactance spéciales ont été placées entre les transformateurs de réglage et le système de barres B, afin de limiter le courant de court-circuit dans ce système. Les transformateurs étant aussi raccordés au systèmè A, on peut alimenter les moteurs des excitatricès par le système à $11 \mathrm{kV}$. dans le cas 'où, pour une raison quelconque, les deux alternateurs auxiliaires se trouveraient immobilisés. Ceci est très important au point de vue sécurité de marche. Cette disposition permettrait également, en cas de nécessité, la mise en marche de la centrale hydraulique par la centrale à vapeur de
Leningrad travaillant en parallèle. Après synchronisation, on pourrait passer à l'auto-excitation. Tous les interrupteurs à huile à 2.200 volts sont manœuvrés électriquement. ceux du système $\mathrm{A}$, de la salle de manœurre et deux du système $\mathrm{B}$, de panneaux de contrôle établis dans la salle de machines. La charpente de l'appareillage à 2.200 volts, en fers cornières, est fermée par du treillis en métal Déployé jusqu'à une hauteur de 2 mètres au-dessus du sol. A l'arrière, se trouvent des portes en métal déployé qui permettent la visite des appareils et du câblage. A l'avant, chaque turbine est pourvue de panneaux amovibles en métal Déployé avec portes, sous les volants des sectionneurs. Tous les sectionneurs sont mancuvrés par perche et munis de contacts de signalisation qui indiquent, dans la salle de

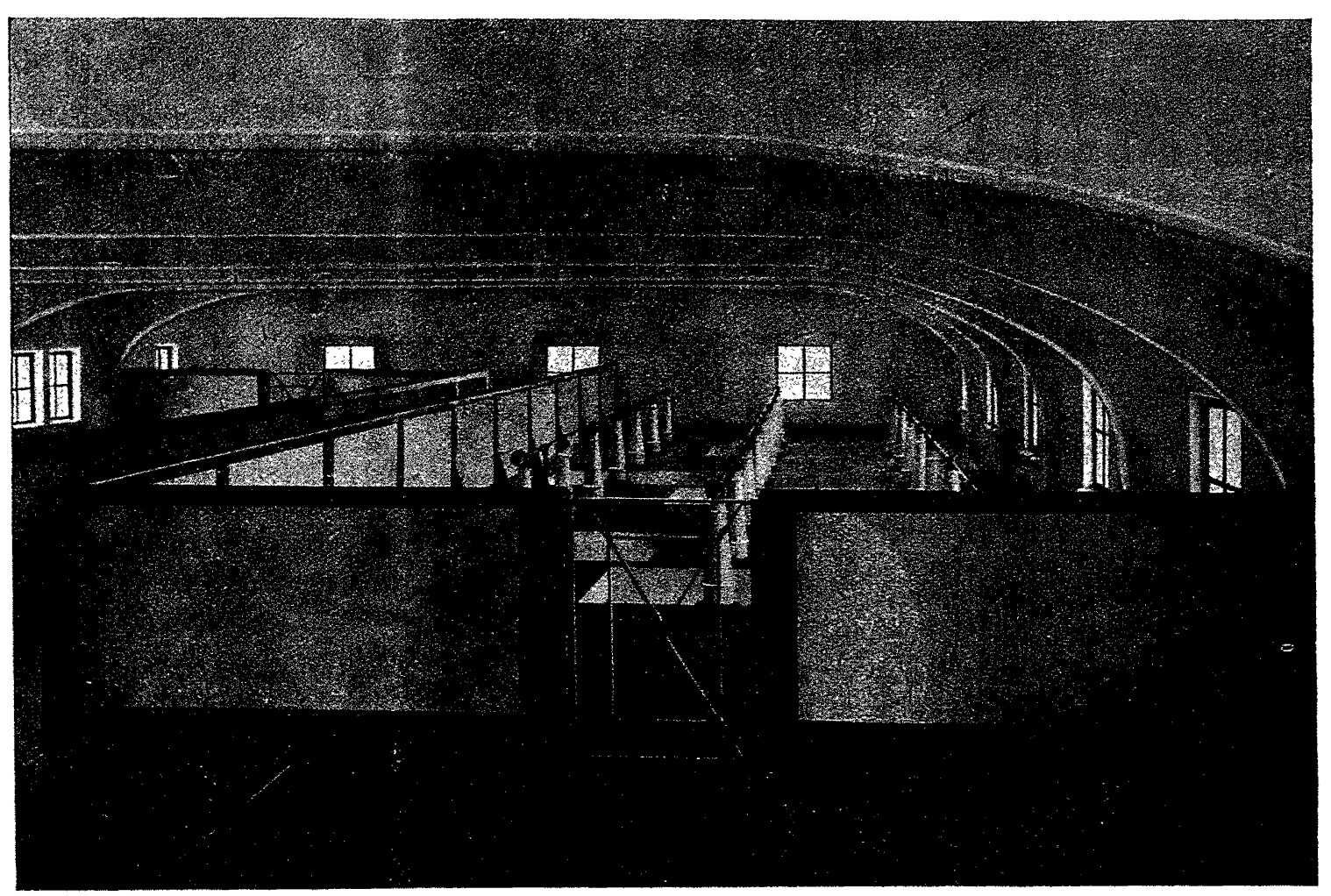

Fig. 9. - Barres omnibus à $120 \mathrm{kV}$.

manœuvre, la position dans laquelle ils se trouvent. Les leviers de manœuvre sont munis de verrous aux positions d'ouverture et de fermeture. Les transformateurs de courant sont du type isolé au compound, ils peuvent supporter un courant de courtcircuit de 5.000 amp. que les disjoncteurs peuvent couper sans aucune difficulté. Ces disjoncteurs sont du type normal $\mathrm{HO}$ tripolaire à cuve unique avec un dispositif de descente du bas pour l'inspection des contacts.

Les panneaux des alternateurs et des transformateurs portent également pour les wattmètres et les synchronoscopes des transformateurs de tension isolés au compound, protégés par des fusibles. Sous les interrupteurs à huile ont été ménagés des bouches d'évacuation d'huile.

Tout le câblage, comme il a été dit, passe par un caniveau encastré dans le plancher. Les deux résistances de terre sont montées contre le mur faisant face au bâtiment des vannes. Comme l'indique le schéma des connexions, des interrupteurs, des sectionneurs et des transformateurs de courant sont insérés dans les circuits de ces résistances.

Le double jeu de barres se trouve installé au-dessus de la charpente d'appareillage, de façon à ce qu'on puisse aisémént déconnecter l'un ou l'autre jeu en vue du nettoyage ou de la visite.

Les communications téléphoniques entre la sous-station de 
Leningrad et la salle de contrôle de la centrale peuvent se faire directement, grâce à un appareil téléphonique à haute tension, couplé en capacité sur la ligne de transmission. Les communications avec la salle des machines se font par un transmetteur d'ordres. Sur un tableau placé à l'extrémité. sud de la salle des machines, on indique au moyen d'un chiffre rouge sur fond bleu, l'unité à laquelle s'applique les instructions données.

Equipement de batterie. Le courant nécessaire au contrôle de la centrale est fourni sous forme de courant continu à 220 volts par une batterie d'accumulateurs qui a une capacité d'environ 540 ampères-heures. L'équipement nécessaire à la charge de la batterie comprend deux groupes générateurs de $31 \mathrm{~kW}$. chacun avec démarreur, tableau de charge et de distribution. La batterie possède en outre un réducteur double, du type rectiligne, qui peut être manœuvré électriquement du panneau de contrôle portant un indicateur de position.

Appareillage des circuits d'alimentation de la centrale. Il y a trois petits tableaux réservés à l'éclairage et aux besoins particuliers de la centrale et combinés avec des transformateurs abaisseurs de tension alimentés par le système auxiliaire à 2.200 volts. L'un de ces tableaux est uniquement réservé à l'éclairage. Il se trouve dans une salle qui donne à l'extrémité sud de la salle de contrôle. Les deux autres sont plạcés dans le couloir qui se trouve au niveau 16,75, sous les gros transformateurs, l'un dans la partie nord, l'autre dans la partie sud de la centrale. Ces tableaux se rapportent aux circuits d'éclairage et en partie à ceux d'alimentation des ateliers, des pompes à huile et à eau, des ascenseurs, etc. Sur tous ces tableaux sont placés des inverseurs automatiques faisant passer automatiquement le circuit d'éclairage de l'alimentation normale en courant alternatif à l'alimentation de secours par batterie, en cas de manque de tension alternative, le retour se faisant automatiquement en cas de reprise de la tension sur le réseau alternatif.

On a installé deux petits panneaux supplémentaires avec transformateurs d'alimentation, pour les trois grues de la centrale. Ces panneaux sont placés respectivement aux extrémités nord et sud et au niveau 30,50. Ils sont alimentés comme les tableaux décrits ci-dessus, par le réseau à 2.200 volts.

Le long des tableaux d'éclairage sont disposés deux jeux d'appareils pour les pompes de régulation. Chaque jeu comporte un transformateur de $100 \mathrm{kVA}$. avec interrupteur à huile, auquel se raccordent les moteurs de pompes, par groupes de deux. Par mesure de sécurité, les trois tableaux d'éclairage et les jeux d'appareils dont nous venons de parler ont été pourvus de commutateurs qui permettent de les raccorder à un transformateur de réserve plus important, qui se trouve placé entre les deux transformateurs des services auxiliaires installés au centre de la centrale.

Pour les essais à haute tension, on a prévu un transformateur d'essai donnant des tensions pouvant atteindre 350.000 volts. Le transformateur est alimenté par un groupe moteur-générateur spécial qui, de même que le transformateur et l'appareillage, est installé dans une petite pièce adjacente à l'atelier de réparation des transformateurs.

Par une traversée murale à 350.000 volts, la tension d'essai est amenée à l'atelier où les dispositions nécessaires ont été prises pour pouvoir procéder à des essais de tension.

Les essais d'huile se font au moyen d'un équipement portatif du type normal Asea avec autotransformateur d'essai à 50.000 volts, appareillage et éclateur noyés dans l'huile.

Mise à la terre. Toutes les charpentes métalliques et tous les supports d'appareils sont soigneusement raccordés à la terre par des câbles de cuivre nus connectés à deux canalisations de terre principales de $120 \mathrm{~mm}^{2}$ de section, quí courent tout le long de la centrale et sont, à leur tour, raccordées à des plarpue: de terre immergées dans le fleuve.

Peinture. Toutes les barres et toutes les connexions son peintes chacune en une couleur qui correspond à la tension es question. Les couleurs adoptées sont les suivantes :

Conducteurs C.A. $>250$ volts : rouge.

Conducteurs C.A. $<250$ volts : gris.

Conducteurs C.C. $\leq 220$ volts : jaune.

Chaque conducteur de phase est, en outre, en certains point convenablement choisis, recouvert, selon la phase à laquelle appartient, d'une bande verte, jaune ou brune.

Ce mode de repérage a été observé dans toute la centrale même pour le petit câblage à l'endroit où il se raccorde aur planchettes à bornes; ce qui simplifie considérablement le essais et les réglages.

Monte-charges. Comme nous l'avons dit, il y a deux grand monte-charges qui servent au, transport des interrupteurs huile à 120.000 volts. Ils ont une capacité de 6 tonnes, qui cor respond au poids de l'un des éléments polaires de l'interrupteur Ils sont placés dans les cages aménagées entre la salle des ma chines et l'appareillage à $120 \mathrm{kV}$., du côté opposé à l'appareillag à $11 \mathrm{kV}$. Le mécanisme de levage, qui comprend un moteu avec interrupteur de fin de course, frein, appareillage de protec tion pour le frein, tambour de câble et poulie de guidage, es placé au niveau 16,75. La poulie guide supérieure est porté par une charpente spéciale, placée immédiatement sous l'étag à $120 \mathrm{kV}$. Les monte-charges desservent trois étages : au niveai 20,50, l'étage du transbordeur de transformateurs de la sall de visite, l'étage des interrupteurs à $120 \mathrm{kV}$. et l'étage des sec tionneurs à $120 \mathrm{kV}$.

Outre ces monte-charges, il y en a encore deux plus petits L'un se trouve dans la partie sud de la centrale; sa capacit maximum est de deux tonnes. Le second est un ascenseur pou le personnel; il est placé au milieu de la centrale, il part d l'étage où se trouve l'appareillage à $2,2 \mathrm{kV}$., traverse la sall des machines et l'étage à $11 \mathrm{kV}$. et va jusqu'à la salle de contrôl avec un arrêt à chaque salle d'appareillage.

C'est un ascenseur à grande vitesse, il est réservé aux ingénieur de la centrale et rend de très grands services.

Résistances de mise en charge. On a installé pour l'essai de alternateurs une résistance spéciale de mise en charge qui été montée au niveau 9,20 à l'extrémité nord de la centrale Cette résistance se compose d'un grand réservoir en béton pourv d'un large trop plein dans lequel on pompe de l'eau.

Les trois électrodes portées par de solides isolateurs du typ extérieur peuvent soit être descendues dans l'eau soit en êtr retirées au moyen d'un appareil de levage commandé électriqu ment du tableau de manœuvre. Pour le raccord aux barres $11 \mathrm{kV}$., on a prévu 5 câbles triphasés de $120 \mathrm{~mm}^{2}$ de sectio qui partent d'une cabine de l'appareillage à $11 \mathrm{kV}$ équipé avec interrupteur à huile et sectionneurs spécialement réservés cet usage. Lą résistance de charge est pourvue d'un châss spécial pour boîte à câble qui porte une boîte munie d'isolateur du type extérieur. Le raccord entre ce châssis et les électrod mobiles se fait par câble flexible en cuivre nu.

Système de protection par relais. Le système de relais a ét étudié de façon à obtenir une sélectivité complète pour to les interrupteurs depuis ceux des alternateurs jusqu'à ceux d lá ligne de transmission dans la sous-station. Il en va de mêm pour le circuit d'alimentation des services auxiliaires de I centrale.

Protection des alternateurs. Le système de protection des alter nateurs comprend : des relais à maxima temporisés à temp constant, des relais différentiels à grande sensibilité combiné 
avec des relais à temps constant à faible temporisation et des relais de signalisation. En cas de surcharge, l'interrupteur à huile de l'alternateur déclenche sous l'action des relais à maxima après la temporisation convenable déterminée d'après les conditions de la sélection. En même temps, le circuit d'excitation s'ouvre au moyen de contacts auxiliaires placés sur l'interrupteur et qui coupent le circuit d'excitation des excitatrices. En cas d'avarie interne, soit dans l'alternateur soit sur ses câbles de connexion, le relais différentiel provoque le déclenchement de l'interrupteur à huile correspondant. A chaque déclenchement automatique de l'interrupteur à huile de l'alternateur, le relais de signalisation entre en action et actionne un signal avertisseur dans la salle de commande, tandis que le déclenchement est automatiquement enregistré sur le tableau de manouvre par un signal indicateur. Les six transformateurs de courant utilisés pour les circuits des relais ont été spécialement construits dans ce but. Ils présentent le maximum de sécurité contre les courts-circuits, alors que les transformateurs de courant utilisés pour les appareils de mesure sont d'une autre construction, spécialement étudiée pour obtenir la plus grande précision possible dans les lectures.

Proteclion des transformateurs. Les transformateurs principaux sont protégés par des relais à maxima à temps constant, des relais différentiels équilibrés combinés avec des relais à temps constant à faible temporisation, des relais auxiliaires de déclenchement instantanés à double contact et des relais de signalisation. Les relais à maxima sont réglés pour une intensité primaire légèrement supérieure au double de l'intensité normale; afin d'empêcher le déclenchement si l'un des transformateurs travaillant en parallèle est mis hors service pour une raison quelconque. Comme il a été dit précédemment, chaque transformateur peut être surchargé sans inconvénient de $100 \%$ pendant un certain temps, ce qui permet la mise en marche du groupe transf̣̂rmateur de réserve avant que le service ait été interrompu par un déclenchement automatique. Les relais à maxima agissent uniquement sur les dispositifs de déclenchement des interrupteurs placés du côté $120 \mathrm{kV}$. des transformateurs. Les relais différentiels, qui agissent lorsque se présente une avarie interne du transformateur lui-même, font déclencher à la fois, par l'intermédiaire des relais auxiliaires instantanés, les interrupteurs à. 120 et à $11 \mathrm{kV}$. du transformateur et isolent ainsi complètement l'appareil avarié. Les relais différentiels sont complètement équilibrés et n'agissent donc pas lorsqu'un courant de circulation traverse les bobinages des relais en cas de court-circuit à l'extérieur du transformateur. D'autre part, l'emploi de relais à temps constant empêche les déclenchements intempestifs dus, par exemple, à des pointes momentanées provoquées par la mise en service des transformateurs. Le relais de signalisation donne l'indication nécessaire dans la salle de commande. Il montre la position dans laquelle se trouvent les interrupteurs et l'action des déclenchements automatiquess.

Protection des deux lignes. Elle comporte des relais à maxima temporisés avec dispositif de verrouillage, des relais wattmétriques directionnels en connexion différentielle, des relais de terre en connexion asymétrique agissant sur des relais à temps constant, des relais de terre ampèremétriques équilibrés, en connexion différentielle actionnant des relais auxiliaires de déclenchement instantanés. Les relais à maxima, pour des raisons semblables à.celles que nous avons indiquées pour les transformateurs, sont réglés pour le double du courant normal et sont liormalement verrouillés au moyen d'un dispositif spécial. En cas de court-circuit sur l'une ou l'autre ligne, le relais wattmétrique directionnel correspondant déconnecte le dispositif de verrouillage du relais à maxima de la ligne qui présente un défaut. Le dispositif de verrouillage de l'autre ligne, au contraire, reste sans changement, ce qui évite tout déclenchement intempestif. Le relais de terre ampèremétrique équilibré appartenant à chacune des lignes coupe instantanément la ligne qui présente un défaut, et la même manœuvre se fait simultanément à la sous-station, de telle sorte que la ligne se trouve complètement isolée. Les relais de terre équilibrés n'agissent pourtant pas dans le cas d'un courant de terre symétrique sur les deux lignes. Dans ce cas, les relais en connexion asymétrique commencent à entrer en action et, après un certain temps, font déclencher les interrupteurs de ligne.

Le dispositif de protection des barres omnibus agit soit en coupant la terre, soit en séparant les sections du système lorsque les deux groupes d'alternateurs travaillent en parallèle. Dans le circuit de terre de chacun des groupes d'alternateurs sont placés des relais à maxima temporisés à temps constant et, en cas de mise à la terre, la connexion de terre du groupe correspondant est coupée après un certain temps. La résistance de terre est calculée pour un courant de terre maximum d'environ $135 \mathrm{amp}$. L'interrupteur de sectionnement peut ainsi isoler directement le système de barres dans lequel se présente le défaut avant que le relais de terre ait eu le temps d'agir.

$\mathrm{En}$ cas de terre sur le système à $11 \mathrm{kV}$. pendant la marche en parallèle des jeux de barres $11 \mathrm{kV}$., une section du système est isolée au moyen du relais de terre ampèremétrique en connexion asymétrique de l'interrupteur de sectionnement, combiné avec un relais auxiliaire instantané; ceci à condition que le courant de terre passe par l'interrupteur de sectionnement.

Les transformateurs des services auxiliaires sont protégés, du côté haute tension, par des relais à maxima temporisés à temps' constant combinés avec des relais auxiliaires de déclenchement instantanés à double contact qui font déclencher à la fois les interrupteurs à huile du côté primaire et du côté secondaire du transformateur. Du côté basse tension, il y a des relais à maxima à temps inverse qui font uniquement déclencher les interrupteurs basse tension.

Les alternateurs auxiliaires et les autres groupes de commande, à l'exception de ceux indiqués ci-dessous, sont protégés par des relais à maxima à temps inverse.

Les moteurs de ventilateurs sont pourvus de relais à maxima à temps constant qui, par conséquent, agissent plus tard que les interrupteurs principaux qui les alimentent. Les interrupteurs en question peuvent ainsi être établis pour une capacité de rupture un peu plus faible.

La synchronisation peut se faire sur le système à $11 \mathrm{kV}$. au moyen des interrupteurs des alternateurs. Les transformateurs de tension nécessaires à la synchronisation sont placés de part et d'autre de chacun des interrupteurs à huile, ce qui empêche toute fausse manœuvre d'enclenchement. Chaque groupe d'alternateurs est pourvu d'un synchronoscope qui, grâce à un commutateur à couteaux pentapolaire commandé de l'avant du panneau de contrôle, peut également êtrę utilisé pour la synchronisation au moyen des interrupteurs de sectionnement.

Excitation et régularisation de tension. Comme il a été dit, chacun des alternateurs est normalement excité par un groupe moteur-générateur qui lui appartient en propre. Ces groupes moteurs-générateurs sont alimentés eux-mêmes par deux groupes moteurs-générateurs dits principaux, un par groupe et chacun contrôlé par un régulateur Tirril pour le réglage automatique de la tension.

Extrait de ASEA-Revue. - Mars-Mai 1929. 